\title{
Problem of close eigenvalues in the vibration testing of structures
}

\author{
V. Gershkovich* N. Haritos* \\ (Received 25 October 2004, revised 8 June 2005)
}

\begin{abstract}
We outline our vibration based testing approach towards reconstruction of structural properties and damage detection of large structures. Our emphasis is on developing algorithms for the detection of close or coinciding eigenvalues and their calculation - a commonly encountered situation that has not been adequately addressed in the modal analysis literature nor in commercial software.
\end{abstract}

\section{Contents}

\section{Introduction}

*Dept. of Civil \& Environmental Engineering, University of Melbourne, Australia. mailto:nharitos@unimelb.edu.au

See http://anziamj.austms.org.au/V46/CTAC2004/Gers for this article, (c) Austral. Mathematical Soc. 2005. Published July 25, 2005. ISSN 1446-8735 
2 Discrete model

C661

3 Separated natural frequencies

C662

4 Close natural frequencies

C664

5 Example - close natural frequencies

C665

6 Separation of coinciding eigenvalues

C667

7 Damage detection

C668

8 Concluding Remarks

C669

References

C670

\section{Introduction}

Small vibration can be used to determine structural integrity or the presence of damage in many fields: aerospace, automotive, civil and mechanical engineering, rotating machinery, medicine, to list just a few [6, 13, e.g.]. Mathematically this is considered to be an inverse problem: reconstruction of coefficients of the wave equation on the basis of measurement of its initial eigenfunctions [5]. Both the mathematical approach adopted and the engineering techniques applied (structure excitation and measurement) vary strongly from field to field. In some fields, like rotating machinery, the problems and methods are simpler, and the situation far more satisfactory [13]. In other fields the problems are more difficult and satisfactory solutions have yet to be achieved.

Our paper relates to one of the "difficult" fields - health monitoring of bridges via experimental modal analysis [8]. The practical importance of the problem is due to safety considerations and that properly developed theory 
and software can result in large savings in the maintenance of a nation's multi-billion dollar road bridge infrastructure asset.

Measurement difficulties arise due to ambient weather conditions, which can affect results. The large size of bridge structures leads to rather extensive experiments that must be well planned in advance to decrease as much as possible the costs of performing them and those associated with closing the bridge to traffic during data acquisition [2].

The problem of close eigenvalues is vital to all steps of damage detection and reconstruction of structural properties. Despite this, there appears to be no investigation of this problem in the literature, and none of the commercial packages adequately deals with it.

Our experience with the practical implementation of these techniques is in the monitoring of structural integrity of road bridges. Close eigenvalues have created problems on a number of occasions, thus motivating the research presented herein. We discuss broad ranging aspects of the problems encountered for closely spaced natural frequencies, develop the necessary elements of the theory and describe numerical methods for their solution.

We consider a scheme in which the excitation force $f_{0} \sin \omega t$ is applied at a single point on the spatial grid of measurement points (in practice a 10 tonne linear hydraulic shaker is used for excitation in bridge applications) with $\omega$ changing inside a range of frequencies $\Omega=\left[\omega_{\min }, \omega_{\max }\right]$. Accelerometers are used for measurement at chosen grid points on the bridge surface from which we calculate bridge displacement under vibration. The scheme consists of two steps: the first step is reconstruction of eigenvalues and eigenfunctions, the second is reconstruction of structural properties and damage detection.

When all natural frequencies are well separated both steps are far simpler to tackle. In the case of closely spaced frequencies it may be possible to miss one of a pair of close natural frequencies and obtain a quasi-mode (linear combination of modes corresponding to close eigenvalues), instead of the actual modes $[1,10]$. Consequently, significant damage may erroneously be 
inferred at the second stage.

We have recently developed algorithms and a software package that effectively detects close or even coincident natural frequencies, when damping values are essentially different. This in turn has allowed us to use perturbation theory to detect and calculate coinciding eigenvalues and their corresponding vector space of eigenmodes.

The advances we have made significantly enhance our ability to perform modal identification studies on vibration data from road bridges for the purpose of structural health monitoring.

\section{Discrete model}

The linear discrete model for small vibration of a structure, $M \ddot{X}+C \dot{X}+$ $K X=f$, is a spatial discretization of the linear wave equation

$$
\left[d^{2} / d t^{2}-L\right] Y=f,
$$

$L$ is a linear elliptic operator, close to a self adjoint operator, and $Y$ is the displacement. In the discrete model, $X$ is the vector of displacements at a properly chosen grid of points on the structure, $M, C$ and $K$ are $n \times n$ matrices respectively of mass, damping and stiffness, and $f(t)=f_{0} e^{i \omega t}$ is an excitation force applied at a point on this grid.

The eigenvalues of (1) form $n$ complex conjugate pairs $\left\{\lambda_{j}=\alpha_{j}+i \beta_{j}, \bar{\lambda}_{j}=\right.$ $\left.\alpha_{j}-i \beta_{j}\right\}_{j=1}^{n}$, where $\beta_{j}$ is the $j$ th natural frequency of $(1), \alpha_{j}<0$, and $-\alpha_{j} \ll$ $\beta_{j}$ relates to the damping of the corresponding mode. We produced $[2,3,4]$ an algorithm and the corresponding software which allows us to find $\lambda_{j}$, $j=1, \ldots, n$, with a proper tolerance, and then to calculate the corresponding modes in the case of essentially separated natural frequencies.

Our method combines the nonlinear least square method with the Newton method and exploits the fact that elements of the matrix $H(\omega)=$ 
$\left(-M \omega^{2}+i \omega C+K\right)^{-1}$ are linear combinations of the fractions $\left(i \omega-\lambda_{j}\right)^{-1}$, $\left(i \omega-\bar{\lambda}_{j}\right)^{-1}, j=1, \ldots, n$ where $H(\omega)=\left\{h_{i, j}(\omega)\right\}$ is the transfer matrix. The coefficients of these linear combination's determine the eigenvectors of (1). To avoid technical details in the discussion to follow, we consider only one of the elements of the transfer matrix and denote this element simply by $h(\omega)$. (In practice we use several such functions simultaneously in our calculations to obtain more precise results [4]). To simplify formulas we denote $\operatorname{frac}(c, \lambda, \omega)=c(i \omega-\lambda)^{-1}+\bar{c}(i \omega-\bar{\lambda})^{-1}$ and $\operatorname{frac}_{j}(\omega)=\operatorname{frac}\left(c_{j}, \lambda_{j}, \omega\right)$. For a simple spectrum, one has an approximation $h(\omega) \approx \sum_{s=1}^{n} \operatorname{frac}_{s}(\omega)[5]$.

\section{Separated natural frequencies}

As a result of measurement we obtain a function $\tilde{h}(\omega)=h(\omega)+\delta(\omega) ; \delta$ is due to measurement errors, "noise", slight non-linearities of the real process, etc. Denote $\mathcal{C}=\left\{c_{1}, \ldots, c_{n}\right\}, \Lambda=\left\{\lambda_{1}, \ldots, \lambda_{n}\right\}$. We define an error function $\operatorname{Err}(\Lambda, \mathcal{C}, \omega)=h(\omega)-\sum_{s=1}^{n} \operatorname{frac}\left(c_{s}, \lambda_{s}, \omega\right)$, denote its $L_{\Omega}^{2}$-norm as $\operatorname{Err}_{2}(\mathcal{C}, \Lambda)$ and its $L_{\Omega}^{\infty}$-norm as $\operatorname{Err}_{\infty}(\mathcal{C}, \Lambda)$. We define $\mathcal{C}_{\text {min }}(\Lambda)$ by the condition $\operatorname{Err}_{2}\left(\Lambda, \mathcal{C}_{\text {min }}\right)=\min _{\mathcal{C}} \operatorname{Err}_{2}(\Lambda, \mathcal{C}), \operatorname{Err}_{2}(\Lambda)=\operatorname{Err}\left(\Lambda, \mathcal{C}_{\text {min }}(\Lambda)\right), \Lambda_{\text {min }}$ by the condition $\operatorname{Err}_{2}\left(\Lambda_{\text {min }}\right)=\min _{\Lambda} \operatorname{Err}_{2}(\Lambda)$, and $\operatorname{Err}_{2}=\operatorname{Err}_{2}\left(\Lambda_{\text {min }}\right)$.

The scheme for calculation was discussed in our previous papers. We summarise it briefly and provide references to further detail.

1. We use multiple measurements and filter the data to decrease $\delta$ and provide convergence of the Newton method and existence of a unique global minimum $\Lambda_{\min }$.

2. The function $\|h\|$ provides an initial approximation $\Lambda_{0}$ for $\Lambda$ min. When all natural frequencies $\beta_{1}<\beta_{2}<\cdots<\beta_{n}$ are well separated, the fraction $\|h(\omega)\| \approx\left\|c_{j}\left(i \omega-\lambda_{j}\right)^{-1}\right\|$ near $\beta_{j} ;\|h\|$ possesses sharp local maxima near each of the natural frequencies $\beta_{j}$, see Figure 1 . One 
can also obtain an initial approximation to $\alpha_{j}$ using $\left\|h\left(\beta_{j}\right)\right\| \approx\left\|c_{j} \alpha_{j}\right\|$, and $\left\|h\left(\beta_{j} \pm \alpha_{j}\right)\right\| \approx\left\|h\left(\beta_{j}\right)\right\| / \sqrt{2}$. These approximations appear to be precise enough to provide convergence in the Newton method.

3. To obtain $\Lambda_{\min }$ we use the Newton method (in fact we have to use a non-homogeneous measure on $\Omega$ in the definition of the $L^{2}$-norm and vary this measure in the calculation process) [4].

4. On each step of the Newton method we calculate $\operatorname{Err}_{2}(\Lambda)$ and its partial derivatives $\partial \operatorname{Err}_{2}(\Lambda) / \partial \lambda_{k}$ using the least square method [4].

5. We use $\Lambda_{\min }$ as an approximation to the eigenvalues and use it together with $\mathcal{C}_{\min }$ to calculate the eigenvectors - this can be done under reasonable assumptions on damping matrix $C$. The reader can find precise formulations and explicit formulas in [3].

6. This leads to reconstruction of matrices $C$ and $K$ in the discrete model. (The matrix $M$ does not change essentially for bridges with any real crack development and can easily be accurately estimated). These matrices well approximate coefficients of the wave equation under conditions of proper measurement planning based on an initial finite element model of the bridge. It involves making a number of decisions:

The choices for the number of natural frequencies to be considered and the frequency range interval $\Omega$ must provide a sufficient number of eigenfunctions to reconstruct the wave equation. (The model situation is reconstruction of the Riemannian metric from several initial eigenfunctions of the Laplacian). Discussion can be found in [5].

The choice of the grid of points for performing response measurements in the structure and the position(s) of the excitation device must provide sufficiently large norms for all nominators $c_{k}$ (to avoid missing a natural frequency in the calculations). Further detail can be found in $[2,5]$. 


\section{Close natural frequencies}

Small vibration of the structure is described by a fourth order elliptic operator $L$. Let us fix a small positive $\epsilon$ and denote by $N_{x, \epsilon}$ the number of natural frequencies of $L$ between $x$ and $x+\epsilon$. The Weyl formula [12, e.g.] gives for large $x: N_{x, \epsilon} \sim \operatorname{Vol} x \epsilon \gg 0$, where $\mathrm{Vol}$ is the volume of structure, under mild assumptions on the structure. Then only a few initial frequencies can be well separated. (The distribution of initial natural frequencies strongly depends on subtle properties of the structure [11]).

When two natural frequencies are very close that is $\Im \lambda_{j} \approx \Im \lambda_{j+1}$, the corresponding local maxima of $\|h\|$ are glued together, see Figure 1. Our strategy is based on the fact that even for very close but different natural frequencies the sum of two corresponding fractions cannot be well approximated by one fraction. What is essential is that these two cases can be distinguished in the presence of noise and measurement errors provided by modern equipment, (under proper measurement procedures described in the previous section and with well developed software). Moreover even for coinciding natural frequencies but essentially different damping, $\Re \lambda_{j}$ and $\Re \lambda_{j+1}$, double natural frequencies can be detected and both eigenvalues can be calculated.

When we suspect double eigenvalues we check the hypothesis that there exists a unique natural frequency near $\beta_{j}=\Im \lambda_{j}$, using the calculation described in the previous section. If Err 2 exceeds significantly the upper bound of possible energy attributed to noise plus modelling errors, we reject the hypothesis. Then we add one more fraction with natural frequency near $\beta_{j}$ and find the best approximation with an extra fraction (we skip technical details).

Denote by $\widetilde{\operatorname{Err}_{2}}, \widetilde{\operatorname{Err}_{\infty}}$ the errors for the best approximation with an extra fraction. Estimates and numerical experiments show that when a fraction is actually missed, the $\widetilde{\operatorname{Err}_{2}}$ is many times smaller than $\operatorname{Err}_{2}$ and $\widetilde{\operatorname{Err}_{\infty}}$ is 


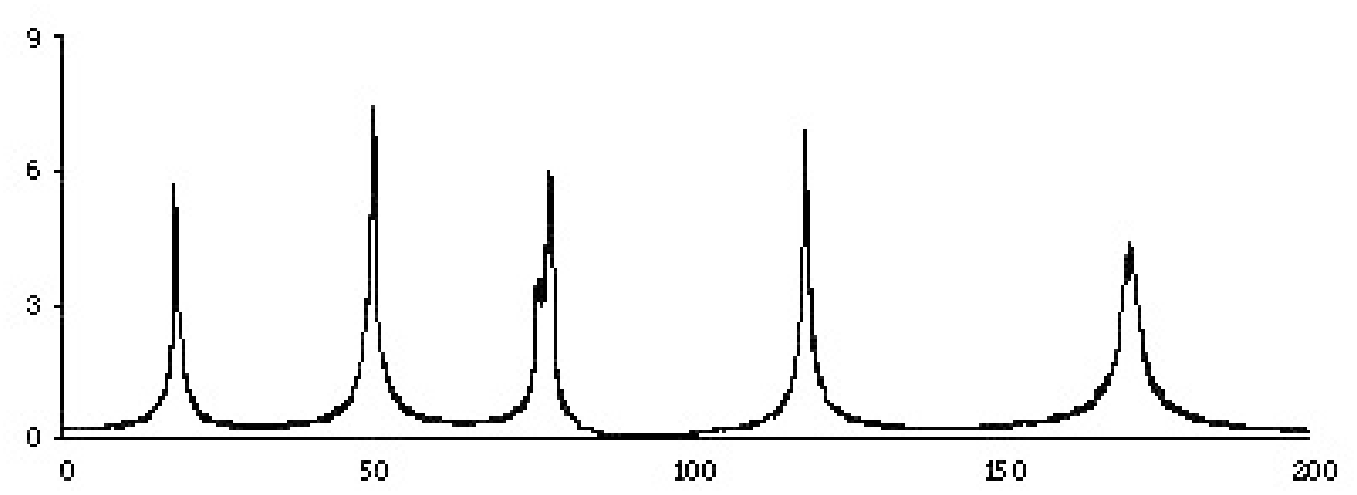

Figure 1: Norm of transfer function $\|h(\omega)\|$

typically tens of times smaller than $\operatorname{Err}_{\infty}$.

We have applied our software for the detection of close natural frequencies to the following example.

\section{Example - close natural frequencies}

We have generated the function $h(\omega)=\sum_{k=1}^{6} \operatorname{frac}_{k}(\omega)+\Upsilon$, where $\Upsilon$ is normally distributed random noise with amplitude up to $3 \%$ of $h(\omega)$, as would typically occur from good quality experimental measurement. We chose artificial but realistic data corresponding closely to the measurement conditions of a real bridge: $\mathcal{C}=(-1.5+1.5 i, 3.05-1.75 i, 3,-2.1+2.4 i, 2.1+$ $2.4 i,-3.7+3.9 i)$ and $\Lambda=(-0.38+18 i,-0.46+49.5 i,-0.6+76 i,-0.45+$ $78 i,-0.45+119 i,-1.2+171 i)$. The function $\|h(\omega)\|$ is presented in Figure 1. The third and fourth natural frequencies are close and the corresponding local maxima of $|h|$ are virtually glued together.

We used our software to reconstruct natural frequencies only from knowledge of $h$. Our hypothesis that there exists only one natural frequency near 


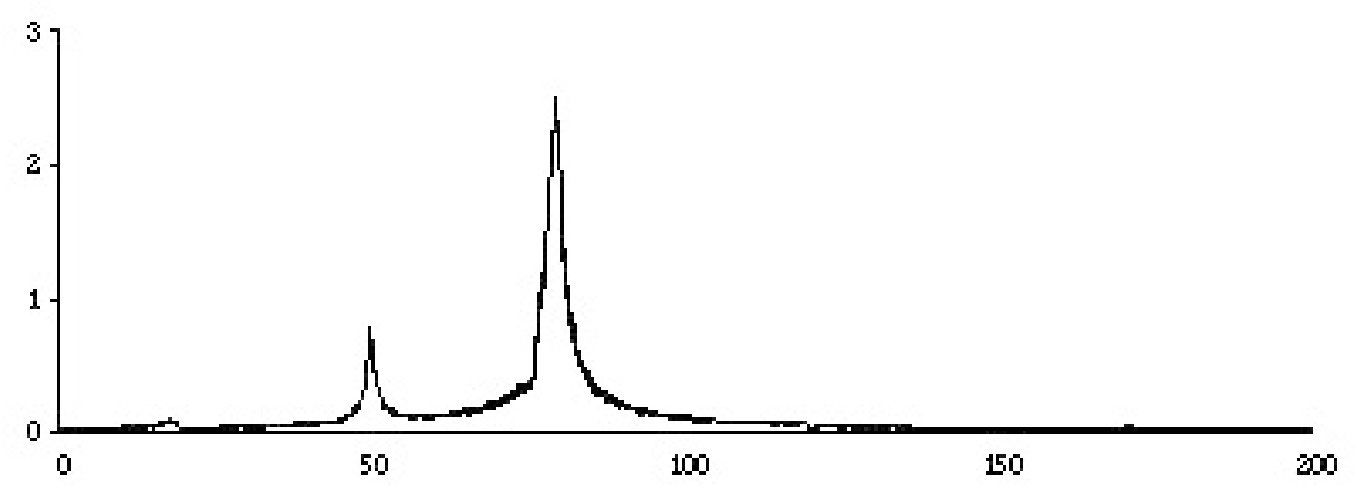

FiguRE 2: Norm of error $\left\|\operatorname{Err}\left(\mathcal{C}_{\min }, \Lambda_{\min }, \omega\right)\right\|$ for the best approximation of $h(\omega)$ when one of the close natural frequencies is missed

$\omega=77$. We construct the best possible approximation to $h$ by the sum of five fractions $h(\omega) \approx \sum_{k=1}^{5} \operatorname{frac}\left(c_{k}, \lambda_{k}, \omega\right)$ with natural frequencies determined by the five local maxima of $\|h\|$. Figure 2 represents a plot of the norm of the error function evaluated using optimization with one fraction missed. The error $\operatorname{Err}\left(\mathcal{C}_{\text {min }}, \Lambda_{\text {min }}, \omega\right)$ in such an optimal approximation exceeds many times the noise level. This result indicates that the conjecture is wrong and that there must exist at least two close natural frequencies near $\omega=77$.

Figure 3 depicts a plot of the error norms after exercising our approach using the correct number of eigenvalues. It is clear that the detection of the closely spaced modes has vastly decreased the error function.

Our software works well even in the case of coinciding natural frequencies if the real parts of the corresponding eigenvalues are essentially different. The case of identically coinciding eigenvalues requires additional effort. 


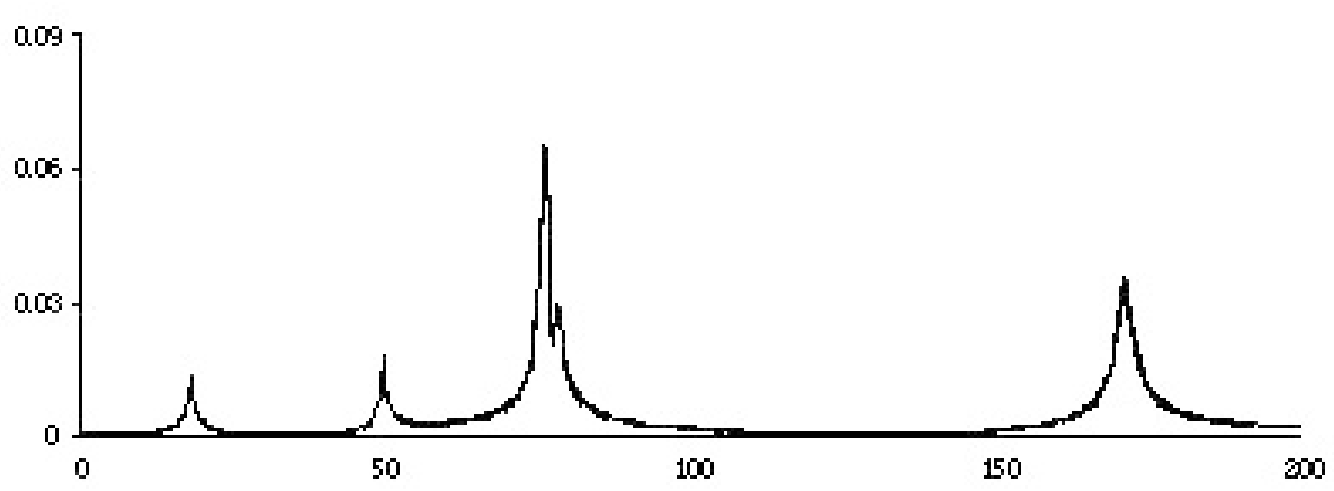

FIgURE 3: Norm of error of the best approximation of $h(\omega)$ when all fractions present

\section{Separation of coinciding eigenvalues}

To detect (almost) coinciding eigenvalues $\lambda_{j} \approx \lambda_{j+1}$, one can physically "perturb" the structure to separate them. We need a perturbation $\delta L$ with a $\left\|\delta \lambda_{j}\right\| \gg\left\|\delta \lambda_{j+1}\right\|$. A simple and rather inexpensive option is to use a heavy concrete block with a small base (on a cart) easily transportable to any point on the bridge.

Let us make a simple calculation. Let $\left\{\lambda_{j}+\delta \lambda_{j}\right\}$ be the spectrum of $L+\delta L$, and $\left\{f_{j}+\delta f_{j}\right\}$ be the set of its eigenfunctions. One can assume that eigenfunctions are orthogonal (but not normalized) and $\left\langle f_{j}, \delta f_{j}\right\rangle=0$ for all $j$. We have $\delta \lambda_{k}=\left\langle(\delta L) f_{k}, f_{k}\right\rangle$ [9]. When our block is over point $x$ of the bridge, we have $\delta \lambda_{k}=\int_{B}(\delta L) f_{k} \bar{f}_{k} d b \approx f_{k}(x) * \int_{B} \delta L d b$ where $B$ is the block base and $d b$ is the corresponding measure.

If we chose a point $x$ such that $f_{j}$ has a local extremum near $x$ and $f_{j+1} \approx 0$, then $\left|\delta \lambda_{j}\right| \gg\left|\delta \lambda_{j+1}\right|$. Note that it is sufficient to take a point $x$ with high accelerometer response when the excitation force has frequency $\omega=\beta_{j}$. Let $\mathcal{Q}$ be the space of quasimodes generated by $f_{j}$ and $f_{j+1}$. Then 
there exists a function $f \in \mathcal{Q}$ such that $|f(x)| \gg 0$. (We take function $g$ orthogonal to $f$ in $\mathcal{Q}$ and apply an orthogonal transformation to obtain $f_{j}$ and $f_{j+1}$ with the required properties).

The function $f_{j+1}$ is very close to the space of quasimodes $\mathcal{Q}$. To obtain a good approximation for the second function we can halve the block mass and take $f_{j}=2 f_{j}^{m / 2}-f_{j}^{m}$, where $f_{j}^{m}$ is the perturbation of $f_{j}$ with mass $m$.

Such a procedure leads to an additional measurement, which can easily be exercised in practice as it does not require (expensive and time consuming) relocation of the shaker. But it is hard to predict "perturbation points" in advance unless one obtains mathematical results related to the "collective behavior" of eigenfunctions. In particular, one has to describe the possible structure of nodal lines and local extrema for a pair of eigenfunctions. Not much is known on this and related topics even for the Laplacians, however see $[7,11]$.

\section{Damage detection}

Damage detection is a fast emerging research field with several concepts and approaches towards its solution to be found in the literature. Two schemes are possible for bridges depending upon whether a single set of measurements is available or multiple sets from periodic testing so that changes can be investigated. Here we present only a brief description related to the latter scheme. Detailed exposition will be the subject of separate publication(s). Intense local damage (such as a crack) leads to strong but local perturbations of (some) of the eigenfunctions. More precisely if we have a strong crack located in a small area $B_{x}$ near an essential local maximum $x$ of an eigenfunction $f$ then perturbation $\delta f$ is essential at points of $B_{x}$ and much smaller (but non-zero) outside $B_{x}$. Perturbation $\delta f$ is much smaller if a crack is near a "nodal point", that is $\|f(x)\| \approx 0$, (compare previous section). 
Note that in the case of coinciding eigenvalues a crack would more strongly affect just one of the eigenvalues and the corresponding eigenfunctions in the crack area. This property allows us to detect a crack even in the case of coinciding eigenvalues.

\section{Concluding Remarks}

Experimental Program. An industrial software package for structural health monitoring must be very reliable and provide correct answers. The first stage of validation of such software is to check "inside" the chosen model. The example we present in this paper is part of such a check. The next step is verification and estimates of the precision of the model and study of nonlinear effects (for example, non-linear boundary conditions due to cracks).

Such a study can be performed only on the basis of well controlled experiments - measurement of structures with known cracks. So far only a small number of such measurements has been performed using data from either bridges before their replacement and/or rather simple artificial structures $[13$, e.g.].

One has to create either a test structure or bridge model which facilitates introduction of temporary "damage" at a given location and of a given extent and intensity. Ideally one should be able to create additional general structure degradation as well as imitate changing weather conditions (for example, strict non-homogeneous temperature change and strong wind).

The substantial potential savings that would result from reliable health monitoring of bridges would justify such an experimental program.

Other Applications. Methods and techniques described in the paper can be applied to other "difficult" and economically important modal analysis 
problems such as the health monitoring of offshore oil rigs. Offshore oil rigs are a complex assemblage of frame members, and pose additional problems in that their mass distribution varies considerably due to operational and environmental influences, for example, storage/removal of oil in tanks, presence or otherwise of marine growth on submerged portions of the primary support structure.

Acknowledgment. The authors are grateful to the anonymous referees for suggestions on improvements to the paper.

\section{References}

[1] Arnold V. Modes and quasi-modes, Functional Analysis and Applications, 6(2):12-20, 1972. C660

[2] Chalko T., Gershkovich V., and Haritos N. Optimal Design of Modal Experiment for Bridge Structures, Proc. XIII Intern. Modal Analysis Conf., (IMAC-XIII), Nashville, p.571-577, 1995. C660, C661, C663

[3] Chalko T., Gershkovich V., and Haritos N. Direct Simulation Modal Approximation Technique, Proc. XIV Intern. Modal Analysis Conf., (IMAC-XIV), Dearborn, p.1130-1136, 1996. C661, C663

[4] Chalko T., Gershkovich V., and Haritos N. Modal Parameter Estimation with Uniform Tolerances, Proc. Intern. Modal Analysis Conf., (IMAC-XV), Tokyo, Japan, p.689-695, 1997. C661, C662, C663

[5] Chalko T., Gershkovich V., and Haritos N. Modal Analysis and Spectral Geometry, Proc. CTAC97, World Scientific, 1:115-122, 1998. C659, C662, C663 
[6] Doebling S. W., Farrar S. R., Prime M. B., and Shevitz D. W., Damage identification and health monitoring of structural and mechanical systems from changes in their vibration characteristics: A literature review, Los Alamos Nat.Lab. Report, LA-13070-MS, 1996. URL C659

[7] Donelly H., Fefferman C. Nodal sets of eigenfunctions on Riemannian manifolds, Inv. Math., 93:161-183, 1988. C668

[8] Ewins D. J. Modal Testing - Theory and Practice, John Wiley, New York, 1985. C659

[9] Kato T. Perturbation theory for linear operators, Springer-Verlag, 1966. C667

[10] Lazutkin V. KAM Theory and Semiclassical Approximations to Eigenfunctions, Springer-Verlag, 1993. C660

[11] Nadirashvili N. Some topics in spectral geometry, Sem. de theorie spectrale et geometrie, Chambery-Grenoble, 9-12, 1990-91. C664, C668

[12] Safarov Y., Vassiliev D. The asymptotic Distribution of Eigenvalues of Partial Differential Operators, Trans. of Math. Monogr., Amer. Math. Soc., 1997. C664

[13] Sohn H., Farrar S. R., Hemez F. N., Shunk D., Stinemates D. W., Nader B. R., A review of structural health monitoring literature 1996-2001, Los Alamos Nat. Lab. Report, LA-13976-MS, 2003. URL C659, C669 